\title{
Influência do sistema de produção e do armazenamento refrigerado nas características físico-químicas e no desenvolvimento de compostos voláteis em morangos
}

\author{
Influence of planting system and cold storage on the physical-chemistries characteristicsand volatile \\ compounds development in strawberries
}

\author{
Julia Maria Machado de Ávila ${ }^{\mathrm{I}}$ Ricardo Peraça Toralles ${ }^{\mathrm{I}}$ Rufino Fernando Flores Cantillano ${ }^{\mathrm{II}}$ \\ Maria do Carmo Ruaro Peralba ${ }^{\text {III }}$ Tânia Mara Pizzolato ${ }^{\text {III }}$
}

\section{RESUMO}

Durante o armazenamento, pode ocorrer a perda de compostos voláteis responsáveis pelo aroma e o aumento de compostos indesejáveis, o que ocasiona a perda de qualidade do morango, reduzindo a vida de prateleira $e$ levando à rejeição do produto pelos consumidores. Neste trabalho, foi avaliada a influência do sistema de produção (convencional e orgânico) e do período de armazenamento $(0,2,5,8$ dias) nas características físicas e químicas $(C F Q) e$ nos compostos voláteis ( $\mathrm{CV}$ ) de morango das cultivares 'Camarosa' e 'Camino Real', utilizando morangos de pomar comercial da região de Pelotas/RS. Os frutos foram colhidos em estádio de maturação comercial e com boa aparência. $O$ planejamento experimental foi casualizado, em triplicata de 15 frutos. Os morangos foram armazenados a $T$ de $1^{\circ} \mathrm{C}$, com 90-95\% umidade relativa (UR). Foram avaliadas a perda de massa, a coloração instrumental [ $L^{*}, a^{*}, b^{*}$ e ângulo hue $\left.(H)\right]$, a firmeza, os sólidos solúveis (SS), a acidez titulável (AT), a relação SS/AT, pH e os teores de CV. Os resultados foram submetidos à análise de variância e as diferenças significativas determinadas pelo teste de Tukey $(P<0,05)$. Os resultados revelaram que $\mathrm{pH}, \mathrm{SS}$, relação $S S / A T$, podridões e perda de massa aumentaram durante o armazenamento. A firmeza se manteve irregular e a AT e $H$ diminuíram significativamente. Os teores de CV aumentaram com exceção do acetato de metila (AM). O etanol (Et), o acetaldeído (AA) e o acetato de etila (AE) correlacionaram-se positivamente com o teor de podridões para a cultivar 'Camino Real' do cultivo orgânico. De modo geral o AM correlacionou-se inversamente com o teor de podridões, exceto para a cultivar 'Camino Real' do cultivo convencional. Os morangos de ambas as cultivares podem ser armazenados por cinco dias com qualidade, independente do sistema de produção.

Palavras-chave: Fragaria $\boldsymbol{x}$ ananassa Duch., produção orgânica, produção convencional, póscolheita, qualidade da fruta.

\section{ABSTRACT}

During the storage a loss of volatile compounds responsible for the aroma and an increase of undesirable compounds can occur leading to a decrease in the strawberry quality, a reduction of the shelf life and product rejection by the consumers. In the present work the influence of the planting system (conventional and organic) and the storage period $(0$, 2, 5 and 8 days) upon physical and chemical characteristics (FCC) as well as on strawberry volatile compounds (VC) in the cultivars 'Camarosa' and 'Camino Real' were evaluated using strawberries from a commercial orchard from Pelotas/RS. The fruits were harvested in a stage of commercial maturation and sound appearance. The experimental planning was randomized, in triplicate of fifteen fruits. The strawberries were stored at $1{ }^{\circ} \mathrm{C}$ and $90-95 \%$ of relative humidity $(R H)$. The mass loss, the instrumental color $\left[L^{*}, a^{*}, b^{*}\right.$ and hue angle $\left.(H)\right]$, the firmness, the soluble solids (SS), the titratable acidity (TA), the SS/TA ratio, the $\mathrm{pH}$ and $\mathrm{VC}$ content were determined. The results were analyzed by analysis of variance and significant differences were determined using Tukey's test $(P<0.05)$. The results show that $\mathrm{pH}$, SS, relation SS/TA, decay and mass loss have increased during storage. The firmness varied irregularly and TA and $H$ decreased significantly. The values of VC increased with the exception of methyl acetate (MA). Ethanol (Et), acetaldehyde (AA) and ethyl acetate (EA) correlated positively with decay for organic cultivated 'Camino Real'. In a general way, the MA correlated inversely with decay with the exception of conventional cultivated 'Camino Real'. Strawberries from both cultures can be stored for five days without loosing quality regardless the production system.

Key words: Fragaria $x$ ananassa Duch., production organic, production conventional, postharvest, fruit quality.

'Departamento de Química, Instituto Federal Sul-Rio-Grandense (IFSUL), Pelotas, RS, Brasil.

"EMBRAPA Clima Temperado, $\mathrm{km}$ 78, BR 392, CP 403, 960001-970, Pelotas, RS, Brasil. E-mail: fernando.cantillano@cpact.embrapa.br. Autor para correspondência.

"IInstituto de Química, Universidade Federal do Rio Grande do Sul (UFRGS), Porto Alegre, RS, Brasil. 


\section{INTRODUÇÃO}

O morango (Fragaria x ananassa Duch.) é mundialmente conhecido e apreciado, sendo que, no Brasil, os estados de Minas Gerais, São Paulo e Rio Grande do Sul (MADAIL, 2008) são os maiores produtores. A produção nacional de morango, tradicionalmente, destina-se ao mercado in natura e à industrialização de geleias, sucos e polpas (CHITARRA \& CHITARRA, 2005; CALVETE et al., 2008), visto que os brasileiros estão mais conscientizados quanto à importância de uma dieta a base de frutas (SPECHT \& BLUME, 2009).

O morango é um fruto não climatérico de curta vida pós-colheita. Sofre processos fisiológicos e físicos, como a respiração e a transpiração, que acarretam mudanças constantes após a colheita, na maioria de caráter irreversível. É um fruto muito suscetível ao ataque de agentes patógenos causadores de podridões (MALGARIM et al., 2006), sendo o aroma e o sabor um resultado de uma complexa interação entre vários composto voláteis $(\mathrm{CV})$ e não-voláteis $(\mathrm{CNV})$ (WATSON et al., 2002). Um sério problema enfrentado pelos produtores são as perdas, por ser um fruto muito perecível, havendo maior necessidade do uso de refrigeração (CANTILLANO et al., 2008). A refrigeração é o sistema mais utilizado na conservação de frutas. Ela retarda, mas não exclui totalmente as alterações ocorridas nas frutas durante o armazenamento, as quais influenciam na qualidade dos morangos in natura, ocasionando a perda de massa fresca, da firmeza, do sabor, do aroma, dos teores de sólidos solúveis (SS), dos CV e das características sensoriais (AMAL et al., 2010; PELAYO-ZALDÍVAR et al., 2005).

Um grande número de (CV) estão presentes em morangos, incluindo ésteres, aldeídos, cetonas, terpenos, alcoois, lactonas e derivados do furano (WATSON et al., 2002). No armazenamento, pode ocorrer perda dos compostos responsáveis pelo aroma e também o acúmulo de produtos indesejáveis e indicadores (acetaldeído (AA), etanol (Et) e acetato de etila (AE)) do desenvolvimento do off-flavor (odor e gosto indesejável) (PÉREZ \& SANZ, 2001). As perdas e/ou acúmulo de produtos indesejáveis ocasionam a perda da qualidade dos morangos, a redução da vida de prateleira e levam à rejeição do produto pelos consumidores.

O objetivo deste trabalho foi estudar a relação entre o sistema de produção (orgânica e convencional) e a influência do período de armazenamento refrigerado nas características físicas e químicas e no desenvolvimento de CV indesejáveis de morango das cultivares 'Camarosa' e 'Camino Real'.

\section{MATERIAL E MÉTODOS}

Foram utilizados morangos (Fragaria $\boldsymbol{x}$ ananassa Duch.) da safra 2008 das cultivares 'Camarosa' e 'Camino Real', produzidos em dois sistemas de produção (convencional e orgânico). Foram colhidos em pomar comercial localizado na estrada da Gama - Monte Bonito, $9^{\circ}$ distrito do município de Pelotas, na latitude $32^{\circ} 52^{\prime} \mathrm{S}$, longitude $52^{\circ} 21^{\prime} \mathrm{W}$ e altitude $220 \mathrm{~m}$. Frutos sem defeitos e de estádio de maturação comercial foram selecionados e armazenados por $0,2,5$ e 8 dias em câmara fria a $1 \pm 0,5^{\circ} \mathrm{C}$ e $90-95 \%$ UR.

As características físicas e químicas (CFQ) foram determinadas no dia da colheita e após cada período de armazenamento, em triplicatas de 15 morangos. Em cada fruto, foram realizadas as análises de perda de massa (PM), cor, firmeza e podridões. A PM (expressa em \%) foi determinada por diferença entre o peso inicial (tempo zero) dos 15 frutos e o de cada período de armazenamento (balança - precisão de $\pm 0,01 \mathrm{~g})$. A leitura de cor $\left(\mathrm{L}, \mathrm{a}^{*}, \mathrm{~b}^{*}\right.$ e ângulo Hue $\left.(\mathrm{H})\right)$ foi determinada por colorímetro Minolta CR-300, com fonte de luz D 65 e $8 \mathrm{~mm}$ de abertura em todos os frutos, sendo a medida determinada com duas leituras em lados opostos na região equatorial. Foi utilizado o padrão C.I.E. para determinação dos valores de $\mathrm{L}^{*} \mathrm{a}^{*} \mathrm{~b}^{*}$, em que os valores de $\mathrm{a}^{*} \mathrm{e} \mathrm{b}^{*}$ foram utilizados para calcular o $\mathrm{H}\left({ }^{\circ} \mathrm{H}^{*}=\operatorname{tang}^{-1} \mathrm{~b}^{*} \cdot \mathrm{a}^{*-1}\right)$. A firmeza expressa em Newton (N) foi medida em duas leituras (igual ao anteriormente citado), utilizando penetrômetro manual modelo FDP 1000 , com ponteira de 5/16 polegadas de diâmetro. Podridões $(\%)$ foram analisadas visualmente nos frutos. Foram considerados podres os que apresentavam sintomas de ataque de patógenos. Os frutos foram moídos em triturador centrífugo (Wallita) para extração do suco e analisados em triplicata. Os teores de acidez titulável (AT), pH e de sólidos solúveis (SS) foram determinados segundo metodologia da AOAC (2000).

A análise dos compostos voláteis (CV) foi adaptada de CANTILLANO (1998). Foram utilizados padrões de AA, Et, AM e AE nas concentrações de 0, $10,25,50$ e $100 \mu \mathrm{g} \mathrm{g}^{-1}$, dissolvidos em água bidestilada. De cada concentração, $30 \mathrm{~mL}$ de solução padrão foram adicionados em frascos lacrados de capacidade de $62 \mathrm{~mL}$ e armazenados a $-18^{\circ} \mathrm{C}$ até a realização das análises cromatográficas. As amostras de suco foram envasadas e armazenadas da mesma forma. Para as análises cromatográficas, as amostras e os padrões foram mantidos em banho-maria por $1 \mathrm{~h}$ a $20^{\circ} \mathrm{C}$, seguido de banho-maria a $60^{\circ} \mathrm{C}$ por uma hora para saturação do espaço livre (Headspace). Uma alíquota de $1 \mathrm{~mL}$ foi retirada do Headspace e injetada em um cromatógrafo 
gasoso Varian 3300 equipado com detector de ionização em chama (DIC) e coluna empacotada ( 6 pés X $1 / 8$ de polegada) de fase Poropak R 80/100 mesh. As condições de operação foram: injector a $175^{\circ} \mathrm{C}$, detector a $200^{\circ} \mathrm{C}$, forno a $150^{\circ} \mathrm{C}$ e $\mathrm{N}_{2}$ como gás de arraste. A identificação dos $\mathrm{CV}$ foi realizada por comparação dos tempos de retenção dos compostos na amostra com os respectivos padrões, e a quantificação por padronização externa.

A PM durante $o$ armazenamento foi corrigida para as seguintes variáveis: SS, AT e CV. Todos os resultados expressos em percentagem foram transformados segundo arco-seno da raiz quadrada para a análise estatística. O software STATISTICA (STASOFT, 1996) foi utilizado para calcular os coeficientes de regressão linear, a análise de variância (ANOVA) e a comparação das médias por Tukey $(\mathrm{P}<0,05)$.

\section{RESULTADOS E DISCUSSÃO}

As cultivares 'Camino Real' e 'Camarosa' nos sistemas de produção orgânico e convencional variaram nas CFQ (Tabelas 1 e 2). Com relação à PM, observou-se uma perda significativa $(\mathrm{P}<0,05)$ e crescente no oitavo dia de armazenamento. A PM foi maior para sistema orgânico frente ao convencional ao longo do período de armazenamento, sendo que a maior perda foi observada na cultivar 'Camino Real' do sistema orgânico no oitavo dia de armazenamento. Tais resultados podem ser comparados ao de NUNES et al. (2005) que relataram uma PM de 11\% para cultivar Oso Grande, sistema convencional, armazenada por oito dias a $1^{\circ} \mathrm{C}$. Segundo HERNANDEZ-MUÑOZ et al. (2006), a perda de massa fresca superior a $10 \%$ é um indicador de perda da qualidade em morangos, ocasionando a rejeição do fruto pelos consumidores. No presente trabalho, considerando o coeficiente de variação, as duas cultivares do sistema convencional apresentaram PM fresca inferior a 10\% no oitavo dia de armazenamento, proporcionando uma melhor conservação e consequentemente melhor aceitação do fruto. NUNES et al. (2005) associaram a perda com a degradação da membrana celular e, por consequência, a liberação da enzima oxidativa polifenoloxidase, que, segundo os autores, foi a principal causadora da degradação das antocianinas, contribuindo para o escurecimento do frutos de morango da cultivar Oso grande durante o armazenamento a $1^{\circ} \mathrm{C}$.

Os valores de $a^{*}$ e $b^{*}$ (valores não apresentados) foram usados para calcular o $(\mathrm{H})$, o qual está relacionado com a cor da epiderme. De um modo geral, observou-se redução do $\mathrm{H}$ durante $\mathrm{o}$ armazenamento (Tabela 1). Na colheita, a cultivar
'Camino Real', produzida no sistema convencional, apresentou menor valor de H $(30,11)$ e " $L^{*}$ " $(30,53)$, indicando que essa cultivar teve uma coloração inicial mais escura do que as demais (Tabela 1). Tal comportamento também foi observado para os outros períodos de armazenamento. O escurecimento acentuado da epiderme do morango durante o armazenamento é um atributo indesejável, tornando o produto pouco aceitável no mercado (CANTILLANO et al., 2008).

Quanto à firmeza, a cultivar 'Camarosa', produzida no sistema orgânico, apresentou valor superior às demais no oitavo dia de armazenamento $(5,36 \mathrm{~N})$. Esse comportamento também foi observado no zero e quinto dia. Já, no segundo dia, a cultivar 'Camino Real', no sistema orgânico (5,43N), foi superior. Com relação ao aumento de firmeza durante o armazenamento, este só foi significativo para as cultivares 'Camino Real' orgânico e 'Camarosa' convencional até o segundo dia. Já para as cultivares 'Camarosa' do sistema orgânico e 'Camino Real' do sistema convencional, não se observaram diferenças estatísticas entre as médias. Segundo PINELI et al. (2008), o aumento de firmeza durante o armazenamento é devido à desidratação ou ressecamento da fruta. Por outro lado, no oitavo dia de armazenamento, observouse decréscimo geral da firmeza, sendo a 'Camino Real' convencional $(2,89 \mathrm{~N})$ significativamente inferior às demais. Segundo SALLATO et al. (2007), o decréscimo de firmeza pode ser devido à proliferação fúngica, causada principalmente por Botrytis cinerea, fato confirmado pelos resultados de maiores valores de podridões no oitavo dia de armazenamento, para as duas cultivares nos dois sistemas de produção (Tabela 1).

$\mathrm{O}$ pH (valores não apresentados na tabela 1) e os SS totais aumentaram no decorrer do armazenamento,sendo maior para o sistema convencional, acompanhado por decréscimo na AT (Tabela 1). Esse comportamento explica o aumento da relação SS/AT (Tabela 1) durante o armazenamento, sendo que, no oitavo dia de armazenamento, a cultivar 'Camino Real' convencional foi significativamente superior $(\mathrm{P}<0,05)$ para relação SS/AT $(13,18)$, seguida da cultivar 'Camarosa' convencional (10,27). Esse índice é considerado um bom indicador para fruta de alta qualidade, quando SS/AT=8,75 (COSTA, 2009). Os valores de $\mathrm{pH}$ no oitavo dia de armazenamento foram significativamente superiores $(\mathrm{P}<0,05)(4,51) \mathrm{e}(4,45)$ para a cultivar 'Camino Real' no sistema de produção convencional e orgânico, respectivamente, enquanto a cultivar 'Camarosa' no sistema orgânico $(4,18)$ apresentou valores significativamente inferior às demais. 
Tabela 1 - Efeito do sistema de produção e do período de armazenamento nas características físico-químicas em cultivares de morango. Safra 2008. Embrapa Clima Temperado, Pelotas, RS, 2011.

\begin{tabular}{|c|c|c|c|c|c|c|}
\hline \multirow{2}{*}{ Variável } & \multirow{2}{*}{ Cultivar/sistema de produção } & \multicolumn{4}{|c|}{ Períodos de armazenamento (dias) } & \multirow{2}{*}{$\mathrm{CV}$ médio $(\%)$} \\
\hline & & 0 & 2 & 5 & 8 & \\
\hline \multirow{4}{*}{ Perda de massa $(\%)$} & CRC & 0,00 & $2,32^{\mathrm{cB}}$ & $2,70^{\mathrm{bB}}$ & $8,93^{\mathrm{cA}}$ & 4,68 \\
\hline & $\mathrm{CC}$ & 0,00 & $2,45^{\text {вв }}$ & $2,60^{\mathrm{bB}}$ & $9,49^{\mathrm{cA}}$ & 3,58 \\
\hline & $\mathrm{CRO}$ & 0,00 & $3,94^{\mathrm{aB}}$ & $3,27^{\mathrm{aC}}$ & $11,21^{\mathrm{aA}}$ & 2,26 \\
\hline & $\mathrm{CO}$ & 0,00 & $2,77^{\text {ьв }}$ & $3,22 \mathrm{aB}$ & $9,70^{\mathrm{bA}}$ & 3,75 \\
\hline \multirow{4}{*}{$\mathrm{L}$} & $\mathrm{CRC}$ & $30,53^{\mathrm{dA}}$ & $28,41^{\mathrm{dB}}$ & $30,40^{\mathrm{cA}}$ & $26,48^{\mathrm{dC}}$ & 1,77 \\
\hline & $\mathrm{CC}$ & $34,40^{\mathrm{bA}}$ & $31,80^{\mathrm{bA}}$ & $30,16^{\mathrm{cA}}$ & $29,66^{\mathrm{bA}}$ & 4,93 \\
\hline & $\mathrm{CRO}$ & $33,83^{\mathrm{cA}}$ & $33,36^{\mathrm{aA}}$ & $31,06^{\mathrm{bA}}$ & $27,08^{\mathrm{cB}}$ & 2,56 \\
\hline & $\mathrm{CO}$ & $36,35^{\mathrm{aA}}$ & $31,30^{\mathrm{cB}}$ & $32,83^{\mathrm{aAB}}$ & $31,24^{\mathrm{aB}}$ & 4,43 \\
\hline \multirow{4}{*}{ Ângulo hue } & $\mathrm{CRC}$ & $30,11^{\mathrm{cA}}$ & $22,16^{\mathrm{cC}}$ & $26,07^{\mathrm{cB}}$ & $24,87^{\mathrm{dBC}}$ & 3,13 \\
\hline & $\mathrm{CC}$ & $33,51^{\mathrm{bA}}$ & $29,14^{\mathrm{bAB}}$ & $26,08^{\mathrm{cB}}$ & $28,96^{\mathrm{bAB}}$ & 5,58 \\
\hline & $\mathrm{CRO}$ & $33,13^{\mathrm{bA}}$ & 30,71 аАв & $27,46^{\mathrm{bBC}}$ & $27,09^{\mathrm{cC}}$ & 3,47 \\
\hline & $\mathrm{CO}$ & $37,28^{\mathrm{aA}}$ & $29,01^{\text {bB }}$ & $29,00^{\mathrm{aB}}$ & $31,93^{\mathrm{aB}}$ & 4,80 \\
\hline \multirow{4}{*}{ Firmeza da polpa (N) } & $\mathrm{CRC}$ & $3,45^{\mathrm{cA}}$ & $3,63^{\mathrm{dA}}$ & $3,66^{\mathrm{dA}}$ & $2,89^{\mathrm{dA}}$ & 11,25 \\
\hline & $\mathrm{CC}$ & $3,04 \mathrm{dC}$ & $4,82^{\mathrm{cA}}$ & $4,44^{\mathrm{bAB}}$ & $3,70^{\mathrm{bBC}}$ & 9,61 \\
\hline & CRO & $3,84^{\mathrm{bB}}$ & $5,43^{\mathrm{aA}}$ & $3,86^{\mathrm{cB}}$ & $3,12^{\mathrm{cB}}$ & 9,40 \\
\hline & $\mathrm{CO}$ & $4,77^{\mathrm{aA}}$ & $5,20^{\mathrm{bA}}$ & $5,40^{\text {aA }}$ & $5,36^{\mathrm{aA}}$ & 11,65 \\
\hline \multirow{4}{*}{ SS (\%) } & $\mathrm{CRC}$ & $5,90^{\mathrm{aB}}$ & $5,93^{\mathrm{aB}}$ & $5,94^{\text {bB }}$ & $6,52^{\mathrm{aA}}$ & 2,82 \\
\hline & $\mathrm{CC}$ & $5,70^{\text {bв }}$ & $5,73^{\mathrm{cB}}$ & $6,21^{\mathrm{aA}}$ & $5,94^{\mathrm{bAB}}$ & 2,04 \\
\hline & CRO & $4,43^{\mathrm{dB}}$ & $4,68^{\mathrm{dAB}}$ & $5,04^{\mathrm{cA}}$ & $4,98^{\mathrm{cA}}$ & 3,02 \\
\hline & $\mathrm{CO}$ & $4,83^{\mathrm{cB}}$ & $5,77^{\mathrm{bA}}$ & $5,78^{\mathrm{bA}}$ & $4,83^{\mathrm{dB}}$ & 1,48 \\
\hline \multirow{4}{*}{ AT $(\%)$} & $\mathrm{CRC}$ & $0,70^{\mathrm{dA}}$ & $0,65^{\mathrm{dA}}$ & $0,69^{\mathrm{bA}}$ & $0,50^{\mathrm{cB}}$ & 3,98 \\
\hline & $\mathrm{CC}$ & $0,79^{\mathrm{bAB}}$ & $0,82^{\mathrm{bA}}$ & $0,68^{\mathrm{cBC}}$ & $0,58^{\mathrm{bC}}$ & 5,00 \\
\hline & $\mathrm{CRO}$ & $0,74^{\mathrm{cA}}$ & $0,76^{\mathrm{cA}}$ & $0,70^{\mathrm{bA}}$ & $0,52^{\mathrm{cB}}$ & 5,25 \\
\hline & $\mathrm{CO}$ & $0,94^{\mathrm{aA}}$ & $0,99^{\mathrm{aA}}$ & $0,95^{\mathrm{aA}}$ & $0,77^{\mathrm{aB}}$ & 3,23 \\
\hline \multirow{4}{*}{ SS/AT } & $\mathrm{CRC}$ & $8,43^{\mathrm{aB}}$ & $9,34^{\mathrm{aB}}$ & $8,60^{\text {bв }}$ & $13,18^{\mathrm{aA}}$ & 6,16 \\
\hline & $\mathrm{CC}$ & $7,96^{\mathrm{aA}}$ & $7,26^{\mathrm{bA}}$ & $9,14^{\text {aA }}$ & $10,27^{\mathrm{bA}}$ & 12,63 \\
\hline & CRO & $6,30^{\mathrm{bB}}$ & $6,14^{\mathrm{cB}}$ & $7,20^{\mathrm{cB}}$ & $9,72^{\mathrm{cA}}$ & 7,40 \\
\hline & $\mathrm{CO}$ & $5,38^{\mathrm{cA}}$ & $6,08^{\mathrm{cA}}$ & $6,18^{\mathrm{dA}}$ & $6,35^{\mathrm{dA}}$ & 10,16 \\
\hline \multirow{4}{*}{ Podridões (\%) } & $\mathrm{CRC}$ & 0,00 & $2,08^{\mathrm{abA}}$ & $1,96^{\mathrm{abA}}$ & $5,88^{\mathrm{abA}}$ & 145,66 \\
\hline & $\mathrm{CC}$ & 0,00 & $12,50^{\mathrm{abA}}$ & $9,80^{\mathrm{ab} A \mathrm{~B}}$ & $12,50^{\mathrm{abA}}$ & 29,36 \\
\hline & $\mathrm{CRO}$ & 0,00 & $0,00^{\text {ьв }}$ & $2,22^{\mathrm{abAB}}$ & $22,55^{\mathrm{aA}}$ & 74,56 \\
\hline & $\mathrm{CO}$ & 0,00 & $10,42^{a b A}$ & $3,93^{\mathrm{abA}}$ & $12,13^{\mathrm{abA}}$ & 66,96 \\
\hline
\end{tabular}

Valores médios seguidos da mesma letra minúscula na coluna e maiúscula na linha não diferem estatisticamente pelo teste Tukey (P>0,05) $(\mathrm{CV}=$ coeficiente de variação, $\mathrm{SS}=$ sólidos solúveis e $\mathrm{AT}=$ acidez titulável $) . \mathrm{CRC}=$ 'Camino Real' convencional; $\mathrm{CC}=$ 'Camarosa' convencional; $\mathrm{CRO}=$ 'Camino Real' orgânico; $\mathrm{CO}=$ 'Camarosa' orgânico.

Na tabela 2, estão apresentados os resultados dos teores de compostos voláteis durante o armazenamento das cultivares de morango 'Camino Real' e 'Camarosa' nos dois sistemas de produção. O acetaldeído (AA) é um volátil que ocorre naturalmente em quase todas as frutas e é acumulado durante o amadurecimento. É precursor do éster etílico produzido durante a maturação de morangos (PESIS, 2005). OAA pode também ser metabolizado para etanol (Et) pela enzima álcool dehidrogenase e para (acetil CoA) pela enzima aldeído dehidrogenase. O Et e a acetil CoA são substratos para a síntese do acetato de etila (AE). AA, 
Tabela 2 - Efeito do sistema de produção e do período de armazenamento nos compostos voláteis das cultivares de morango. Safra 2008. Embrapa Clima Temperado, Pelotas, RS, 2011.

\begin{tabular}{|c|c|c|c|c|c|c|}
\hline \multirow{2}{*}{ Variável } & \multirow{2}{*}{ Cultivar/ sistema de produção } & \multicolumn{4}{|c|}{ Períodos de armazenamento (dias) } & \multirow{2}{*}{$\begin{array}{c}\text { CV médio } \\
(\%)\end{array}$} \\
\hline & & 0 & 2 & 5 & 8 & \\
\hline \multirow{4}{*}{ Acetaldeído (AA) } & CRC & $7,70^{\mathrm{cD}}$ & $7,89^{\mathrm{cC}}$ & $11,81^{\mathrm{aB}}$ & $14,10^{\mathrm{bA}}$ & 0,42 \\
\hline & $\mathrm{CC}$ & $9,27^{\mathrm{aC}}$ & $7,76^{\mathrm{dD}}$ & $9,60^{\mathrm{cB}}$ & $14,34^{\text {aA }}$ & 0,54 \\
\hline & $\mathrm{CRO}$ & $6,94 \mathrm{dD}$ & $8,09^{\mathrm{bC}}$ & $10,05^{\mathrm{bB}}$ & $13,99^{\mathrm{bA}}$ & 0,26 \\
\hline & $\mathrm{CO}$ & $8,53^{\mathrm{bC}}$ & $9,65^{\mathrm{aB}}$ & $8,36^{\mathrm{dD}}$ & $10,72^{\mathrm{cA}}$ & 0,54 \\
\hline \multirow{4}{*}{ Etanol (Et) } & $\mathrm{CRC}$ & $33,18^{\mathrm{cB}}$ & $19,22^{\mathrm{bC}}$ & $33,86^{\mathrm{aB}}$ & $45,73^{\mathrm{bA}}$ & 1,19 \\
\hline & $\mathrm{CC}$ & $46,78^{\mathrm{bA}}$ & $11,56^{\mathrm{dD}}$ & $20,62^{\mathrm{cC}}$ & $42,12^{\mathrm{cB}}$ & 1,25 \\
\hline & $\mathrm{CRO}$ & $28,11^{\mathrm{dB}}$ & $22,72^{\mathrm{aC}}$ & $22,94^{\mathrm{bC}}$ & $48,52^{\mathrm{aA}}$ & 1,45 \\
\hline & $\mathrm{CO}$ & $59,06^{\mathrm{aA}}$ & $17,66^{\mathrm{CC}}$ & $14,61 \mathrm{dD}$ & $35,56^{\mathrm{dB}}$ & 0,64 \\
\hline \multirow{4}{*}{ Acetato de metila(AM) } & $\mathrm{CRC}$ & $7,27^{\mathrm{cA}}$ & $6,77^{\mathrm{cB}}$ & $5,76^{\mathrm{cC}}$ & $4,51 \mathrm{dD}$ & 0,56 \\
\hline & $\mathrm{CC}$ & $15,88^{\mathrm{aA}}$ & $8,67^{\text {bB }}$ & $8,19^{\mathrm{bC}}$ & $8,13^{\mathrm{aC}}$ & 0,78 \\
\hline & $\mathrm{CRO}$ & $6,62^{\mathrm{dA}}$ & $5,95 \mathrm{~dB}$ & $5,08^{\mathrm{dC}}$ & $4,95^{\mathrm{cD}}$ & 0,62 \\
\hline & $\mathrm{CO}$ & $13,43^{\mathrm{bA}}$ & $11,24 \mathrm{aB}$ & $9,48^{\mathrm{aC}}$ & $7,09^{\mathrm{bD}}$ & 0,34 \\
\hline \multirow{4}{*}{ Acetato de etila (AE) } & $\mathrm{CRC}$ & $6,12^{\mathrm{cC}}$ & $5,94^{\mathrm{bD}}$ & $7,12^{\mathrm{cB}}$ & $19,23^{\mathrm{cA}}$ & 0,47 \\
\hline & $\mathrm{CC}$ & $20,62^{a B}$ & $6,16^{\mathrm{bD}}$ & $16,68^{\mathrm{aC}}$ & $45,94^{\mathrm{aA}}$ & 0,35 \\
\hline & $\mathrm{CRO}$ & $5,63 \mathrm{dC}$ & $4,04^{\mathrm{cD}}$ & $6,00^{\mathrm{dB}}$ & $16,23^{\mathrm{dA}}$ & 0,38 \\
\hline & $\mathrm{CO}$ & $11,89^{\mathrm{bB}}$ & $8,06^{\mathrm{aD}}$ & $8,22^{\mathrm{bC}}$ & $21,65^{\mathrm{bA}}$ & 0,30 \\
\hline
\end{tabular}

*Valores médios $\left(\mu \mathrm{g} \mathrm{g}^{-1}\right)$ seguidos da mesma letra minúscula na coluna e maiúscula na linha não diferem estatisticamente pelo teste Tukey $(\mathrm{P}>0,05)(\mathrm{CV}=$ coeficiente de variação) .

Et e AE são produzidos por morangos quando estocados sob condições aeróbicas (ALMENAR et al., 2009). Observou-se aumento na concentração de AA para as cultivares 'Camino Real' e 'Camarosa' no sistema convencional e orgânico, quando armazenadas por oito dias a $1 \pm 0,5^{\circ} \mathrm{C}$. De modo geral, esse aumento foi observado entre o quinto e oitavo dia para o etanol e o acetato de etila. Esses resultados concordam com os de ALMENAR et al. (2009), que relataram, para cultivar 'Camarosa', uma evolução progressiva na concentração de AA, Et e AE após sete dias de armazenamento a $10^{\circ} \mathrm{C}$, com aplicação de 1 a $1,5 \%$ de quitosana. A acumulação de AA e do Et durante o armazenamento sob condições aeróbicas, segundo ALMENAR et al. (2009), está associada à maturação bem como à infecção microbiana causada por danos ao fruto. Por outro lado, no presente trabalho, observou-se decréscimo gradativo do AM para as duas cultivares nos dois sistemas de produção. Para o AA, os maiores valores foram observados para as cultivares 'Camarosa' e 'Camino Real' convencional $\left(14,34 \mu \mathrm{g} \mathrm{g}^{-1}\right.$ e $14,10 \mu \mathrm{g} \mathrm{g}^{-1}$, respectivamente) e 'Camino Real' orgânico $\left(13,99 \mu \mathrm{g} \mathrm{g}^{-1}\right)$ no oitavo dia de armazenamento. Para o Et, a cultivar 'Camarosa' orgânico $\left(59,06 \mu \mathrm{g} \mathrm{g}^{-1}\right)$ foi significativamente superior às demais $(\mathrm{P}<0,05)$ com zero dia de armazenamento a $1^{\circ} \mathrm{C}$. Observou-se uma correlação negativa entre podridões e AM (Tabela 3) para as duas cultivares no sistema de produção orgânico e para a cultivar 'Camarosa' no sistema convencional, indicando que $\mathrm{AM}$ não é metabólito da fermentação. Nos teores de AA, a correlação foi positiva somente para as cultivares do sistema orgânico CRO ( $r=0,82, \mathrm{P}<0,05)$ e $\mathrm{CO}(\mathrm{r}=0,63, \mathrm{P}<0,05)$. Para as concentrações de ET e AE, a correlação com as podridões foram positivas somente para a cultivar 'Camino Real' do cultivo orgânico ( $r=0,80, \mathrm{P}<0,05)$ e ( $\mathrm{r}=0,84, \mathrm{P}<0,05)$. Além disso, a cultivar 'Camino Real' do sistema orgânico apresentou maior podridão e foi a única cultivar com correlação positiva para AA, Et e $\mathrm{AE}$, mostrando que o aumento desses metabólitos é um bom indicador não só de fermentação, mas também de podridão.

\section{CONCLUSÃO}

Os resultados obtidos indicaram que houve aumento de podridões, $\mathrm{pH}$, sólidos solúveis, relação sólidos solúveis/acidez titulável, perda de massa, concentração dos voláteis acetaldeido, etanol e acetato de etila, enquanto que a acidez titulável e o ângulo de Hue diminuíram significativamente, bem como a concentração do volátil acetato de metila ao longo do período de armazenamento por oito dias a $1^{\circ} \mathrm{C}$. Os voláteis etanol, acetaldeído e acetato de etila correlacionaram-se positivamente com a podridão para a cultivar 'Camino Real' do cultivo orgânico, indicando que tais metabólitos podem ser indicadores de fermentação e de podridão. Comportamento oposto foi apresentado pelo acetato de metila, para quase todas as cultivares, exceto para o 'Camino Real' convencional. 
Tabela 3 - Coeficientes de correlação entre podridões e os compostos voláteis para as cultivares de morangos produzidas nos sistemas de produção convencional e orgânico, armazenados durante 8 dias. Safra 2008. Embrapa Clima Temperado, Pelotas, RS, 2011.

\begin{tabular}{|c|c|c|c|c|}
\hline & $\mathrm{CRC}$ & $\mathrm{CC}$ & CRO & $\mathrm{CO}$ \\
\hline Acetaldeído (AA) & $0,45^{\mathrm{ns}}$ & $0,24^{\mathrm{ns}}$ & $0,82^{*}$ & $0,63^{*}$ \\
\hline Etanol (Et) & $0,29^{\mathrm{ns}}$ & $-0,49^{\mathrm{ns}}$ & $0,80^{*}$ & $-0,42^{\mathrm{ns}}$ \\
\hline Acetato de metila (AM) & $-0,50^{\mathrm{ns}}$ & $-0,80^{*}$ & $-0,63^{*}$ & $-0,60^{*}$ \\
\hline Acetato de etila (AE) & $0,48^{\mathrm{ns}}$ & $0,11^{\mathrm{ns}}$ & $0,84^{*}$ & $0,33^{\mathrm{ns}}$ \\
\hline
\end{tabular}

" significativo a $\mathrm{P} \leq 0,05 ; \mathrm{ns}=$ não significativo; $\mathrm{CRC}=$ 'Camino Real' convencional;

$\mathrm{CC}=$ 'Camarosa' convencional; $\mathrm{CRO}=$ 'Camino Real' orgânico; $\mathrm{CO}=$ 'Camarosa' orgânico.

Também foi verificado que os morangos de ambas as cultivares podem ser armazenados sem perda da qualidade por período de cinco dias a $1{ }^{\circ} \mathrm{C}$, independente do sistema de produção.

\section{.REFERÊNCIAS}

AMAL, S.H.A. et al. Improving strawberry fruit storability by edible coating as a carrier of thimol or calcium chloride. Journal of Horticultural Science \& Ornamental Plants, v.2, n.3, p.88-97, 2010. Disponível em: <http://idosi.org/jhsop/2(3)10/ 2.pdf>. Acesso em: 29 jan. 2012.

AOAC (OFFICIAL METHODS OF ANALYSIS OF AOAC) International/Willian Horwitz (Ed.). 17.ed. Maryland, 2000. $1018 \mathrm{p}$.

ALMENAR, E. et al. Evolution of selected volatiles in chitosancoated strawberries (Fragaria x ananassa) during refrigerated storage. Journal of Agricultural and Food Chemistry, v.57, n.3, p.974-980, 2009. Disponível em: <http:// pubs.acs.org/doi/abs/10.1021/jf802319v>. Acesso em: 26 jun. 2010. doi: $10.1021 / \mathrm{jf} 802319 \mathrm{v}$.

CAlvete, E.O. et al. Fenologia, produção e teor de antocianinas de cultivares de morangueiro em ambiente protegido. Revista Brasileira de Fruticultura, v.30, p.396401, 2008. Disponível em: <http://www.scielo.br/ scielo.php?pid=S010029452008000200022\&script=sci_arttext $>$. Acesso em: 06 fev 2012. doi: 10.1590/S010029452008000200022.

CANTILLANO, R.F.F. Estudio del efecto de las atmósferas modificadas durante el almacenamiento y comercialización de algunas frutas y hortalizas, 1998. 275f. Tese (Doutorado em Tecnologia de Alimentos) Universidad Politécnica de Valencia, UPV, Espanha.

CANTILLANO, R.F.F. et al. Qualidade físico-química e sensorial de cultivares de morango durante o armazenamento refrigerado, Embrapa Clima Temperado. Boletim de Pesquisa e Desenvolvimento, v.75, p.1-31, 2008.

CHITARRA, M.I.F.; CHITARRA, A.B. Pós-colheita de frutos e hortaliças: fisiologia e manuseio. 2.ed.rev. e ampl. Lavras: UFLA, 2005. 785p.
COSTA, F.B. Fisiologia e conservação de cultivares de morangos inteiros e minimamente processado. 2009. 115f. Tese (Doutorado em Fisiologia Vegetal) - Curso de Pósgraduação em Fisiologia Vegetal, Universidade Federal de Viçosa, MG.

HERNANDEZ-MUÑOZ, P. et al. Effect of calcium dips and chitosan coatings on postharvest life of strawberries (Fragaria $\boldsymbol{x}$ ananassa). Postharvest Biology and Technology, v.39, p.247-253, 2006. Disponível em: <http://pubs.acs.org/doi/abs/ 10.1021/jf00054a014>. Acesso em: 22 fev. 2009. doi: $10.1021 / \mathrm{jf00054a014.}$

MADAIL. J.C.M.A. Economia do morango. In: SIMPÓSIO NACIONAL DO MORANGO, 4.; ENCONTRO DE PEQUENAS FRUTAS E FRUTAS NATIVAS DO MERCOSUL, 3., Pelotas, RS. Palestras e Resumos... Pelotas: Embrapa Clima Temperado, 2008. p.15-20.

MALGARIM, M.B. et al. Sistemas e condições de colheita e armazenamento na qualidade de morangos cv. 'Camarosa'. Revista Brasileira de Fruticultura, v.28, n.2, p.185-189, 2006. Disponível em: <http://www.scielo.br/ scielo.php?script=sci_arttext\&pid=S010029452006000200007>. Acesso em: 27 out. 2009. doi: 10.1590/S010029452006000200007.

NUNES, M.C.N. et al. Possible influences of water loss and polyphenol oxidase activity on anthocyanim content and discoloration in fresh ripe strawberry (cv. 'Oso grande') during storage at $1{ }^{\circ} \mathrm{C}$. Journal of Food Science, v.70, n.1, p.79-84, 2005. Disponível em: <http://onlinelibrary.wiley.com/doi/ 10.1111/j.1365-2621.2005.tb09069.x/abstract>. Acesso em: 14 fev. 2009. doi: 10.1111/j.1365-2621.2005.tb09069.x.

PELAYO-ZALDÍVAR, C.P. et al. Cultivar and harvest date effects on flavor and other quality attributes of california strawberries. Journal of Food Quality, v.28, n.1, p.78-97, 2005. Disponível em: <http://onlinelibrary.wiley.com/doi/ 10.1111/j.17454557.2005.00005.x/abstract>. Acesso em: 22 maio, 2010. doi: 10.1111/j.1745-4557.2005.00005.x/.

PÉREZ, A.G.; SANZ, C. Effect of high-oxygen and highcarbondioxide atmospheres on strawberry flavour and other quality traits. Journal of Agricultural and Food Chemistry, v.49, p.2370-2375, 2001. Disponível em: <http://pubs.acs.org/ doi/abs/10.1021/jf0014381>. Acesso em: 03 jun. 2010. doi: 10.1021/jf0014381.

Ciência Rural, v.42, n.12, dez, 2012. 
PESIS E. The role of the anaerobic metabolites, acetaldehyde and ethanol, in fruit ripening, enhancement of fruit quality and fruit deterioration. Postharvest Biology and Technology, v.37, n.1, p.1-19, 2005. Disponível em: <http:// www.sciencedirect.com/science/article/pi i/ S0925521405000608>. Acesso em: 28fev.2012. doi: 10.1016/ j.postharvbio.2005.

PINELI, L.L.O. et al. Caracterização física, química e sensorial de morangos 'Osogrande' e 'Camino Real' armazenados a 5 e a $15^{\circ} \mathrm{C}$. In: CONGRESSO BRASILEIRO DE OLERICULTURA, 48., 2008, Maringá/PR. Resumo... ABH p.S5497-S5503(CD -ROM). Disponível em: 〈www.abhorticultura.com.br/〉. Acesso em: 03 jun 2010.

SPECHT, S.; BLUME, R. Competitividade e segmento de mercado à cadeia do morango: algumas evidências sobre o panorama mundial e brasileiro. In: SOBER - SOCIEDADE BRASILEIRA DE ECONOMIA, ADMINISTRAÇÃO E SOCIOLOGIA RURAL, 47., 2009, Porto Alegre, RS. Anais... Porto Alegre: SOBER. 2009. p.1-17.
SALLATO, B.V. et al. Effect of boscalid on postharvest decay of strawberry caused by Botrytis cinerea and Rhizopus stolonifer. Spanish Journal of Agricultural Research, v.5, n.1, p.6778, 2007. Disponível em: 〈http://scholar.google.com.br/〉. Acesso em: 03 fev. 2012.

STASOFT, Inc. STATISTICA for Windows (Computer program manual), 1996. Tolsa, OK: StatSoft, Inc., 2300 East 14 th Street, Tulsa, OK 74104, phone: (918) 749-1119, fax: (918) 749-2219, e-mail: info@statsoftinc.com. Disponível em: <http: //www.statsoftinc.com>. Acesso em: 09 mar. 2009.

WATSON, R. et al. Influence of harvest date and light integral on the development of strawberry flavour compounds. Journal of Experimental Botany, v.53, n.377, p.2121-2129, 2002. Disponível em: <http://jxb.oxfordjournals.org/content/53/377/ 2121.abstract>. Acesso em: 21 maio, 2010. doi: 10.1093/jxb/ erf088. 